\title{
DINÁMICAS Y CAUSAS DE DEFORESTACIÓN EN BOSQUES DE LATINO AMÉRICA: UNA REVISIÓN DESDE 1990
}

\author{
Forest deforestation dynamics and drivers in Latin America: \\ a review since 1990
}

Palabras clave: América Latina, cobertura forestal, pérdida de bosques, tasas de cambio, trópico.

Key words: Latin America, forest cover, forest loss, change rates, tropical.

\section{RESUMEN}

Durante los últimos años se han publicado varios estudios sobre deforestación en Latino América, donde las tasas de pérdida de bosques, hasta la década del año 2000 en la región, por lo general son inferiores a las de otras zonas tropicales. Regularmente, los estudios de deforestación son regionales o estatales o a nivel de cuenca o microcuenca, y no permiten observar la variabilidad intrarregional presente en los trópicos americanos. En este trabajo se presenta el resultado de un metaanálisis de 283 artículos indexados sobre pérdida de cobertura forestal para diferentes tipos de bosques en América Latina (Atlánticos, Montanos, Secos, de Tierras bajas y otros), desde el año 1990 hasta el 2012, así como los factores identificados como causantes de deforestación. Con una tasa general de deforestación de -1.54 para la región, los resultados indican que se presenta una alta variabilidad por países, e incluso se encuentran casos de ganancias de cobertura forestal como en El Salvador. Los bosques más afectados y con tasas de cambio mayores son los bosques secos, seguidos por los bosques montanos. La mayoría

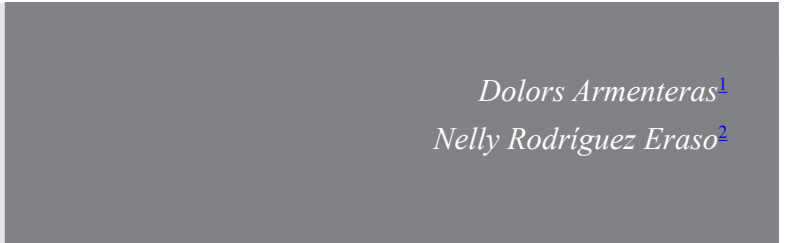

de los países identifica la expansión agrícola y ganadera como la principal causa de deforestación.

\begin{abstract}
Over the past few years there have been a considerable number of studies on deforestation in Latin America. Deforestation rates reported up to the 2000s are generally lower in the region than in other tropical areas. The causes of deforestation in Latin America are similar to those identified in other regions. In general, studies of deforestation are regional or very localized and do not permit comparison of intraregional variability within the American tropics. In this paper we present results obtained from a meta-analysis of 283 articles on deforestation rates for different types of forests in Latin America (Atlantic, Montane , Dry, Lowland and others). Causes of deforestation identified in the literature and published at the national or subnational level since 1990 are also analyzed. There is an overall deforestation rate of -1.54 for the region, but results indicate a high variability of deforestation rates between countries and that there are even cases of forest cover gains, e.g. in El
\end{abstract}

${ }^{1}$ Grupo de Ecología del Paisaje y Modelación de Ecosistemas, Departamento de Biología. Universidad Nacional de Colombia, Bogotá, Colombia. Autor para correspondencia. darmenterasp@unal.edu.co

${ }^{2}$ Grupo de Ecología del Paisaje y Modelación de Ecosistemas, Departamento de Biología. Universidad Nacional de Colombia, Bogotá, Colombia. neraso2000@gmail.com

http://dx.doi.org/10.14483/udistrital.jour.colomb.for.2014.2.a07

Para citar este artículo: Armenteras D. Rodríguez Eraso N. (2014) Dinámicas y causas de deforestación en bosques de latino américa: una revisión desde 1990. Colombia Forestal, 17(2), 233-246. 
Salvador. The highest deforestation rates are in dry forest followed by montane forests. Most countries identify agricultural and livestock expansion as the main cause of deforestation.

\section{INTRODUCCIÓN}

La destrucción de los bosques tropicales ha recibido atención mundial debido a que estos ecosistemas tienen un papel único en términos ecológicos, la diversidad de funciones que proveen $\mathrm{y}$, sobretodo, la incesante amenaza a su existencia, con efectos directos sobre las emisiones netas de carbono debidas a la deforestación y degradación (Houghton, 2012). A esto se suma que, las tasas de deforestación están lejos de ser uniformes en todo el mundo y dependen de los diferentes análisis y fuentes de datos usados para su cálculo. FAO (2011) estima una deforestación neta a nivel mundial de $0.20 \%$ en la década que va de 1990 a $2000,0.12 \%$ entre el 2000 y el 2005 y $0.14 \%$ entre el 2005 y el 2010, con una pérdida neta de 5.2 millones de hectáreas en la década del 2000 al 2010; mientras que Hansen et al., (2010) indican un tasa de $0.6 \%$ anual, con una superficie estimada de pérdida de bosques mundial, entre el $2000 \mathrm{y}$ el 2005 de $1011000 \mathrm{~km}^{2}$, donde cerca de 3.9 millones de $\mathrm{km} 2$ se asocian a procesos de aprovechamiento forestal por tala selectiva de madera (Asner et al. $\underline{2009)}$.

A nivel continental, Achard et al. (2002) reportaron una tasa de deforestación de $0.38 \%$ para América Latina, $0.43 \%$ para África, $0.91 \%$ para el Sudeste de Asia y una tasa global de $0.52 \%$. Brasil e Indonesia representaban el $20.3 \%$ de la pérdida de bosques tropicales en 1980, el $25.7 \%$ de la pérdida durante la década de 1990 y el $40.7 \%$ de la pérdida entre el 2000 y el 2005; sin embargo en el último periodo dichas regiones han reducido significativamente su tasa, mientras que en Australia la sequía y los incendios han acelerado la pérdida de bosques desde el 2000 (FRA, 2010). En la década de los noventa algunos autores estiman una disminución en la tasa de deforestación y un aumento en el área de bosques a través de plantaciones o expansión natural y recuperación de los bosques existentes

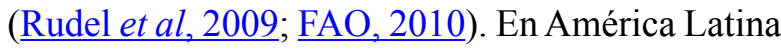

la mayor pérdida neta de bosques en las últimas décadas se presentó en la década del 2000 al 2010 (4 millones ha/año), con un aumento en el periodo del 2000 al 2005 (타, 2010).

Paralelo a los reportes de las tasas de deforestación, es una prioridad global desde los años ochenta comprender las dinámicas humanas $\mathrm{y}$ las consecuencias ambientales en diferentes escalas. Así, a pesar de las claras limitaciones existentes en términos de información homogénea, tanto en lo social como en lo ambiental, existen avances importantes en cuanto a la explicación de las causas de los patrones de deforestación en el trópico (Geist \& Lambin 2001). Inicialmente se avanzó más en términos conceptuales, planteándose dos caminos divergentes: la causalidad debida a un solo factor (generalmente asociado con el crecimiento poblacional), frente a la causalidad derivada de la interacción y complejidad de factores subyacentes. Sumado a lo anterior, la expansión agrícola en zonas de frontera forestal es hoy en día probablemente la causa más citada en la literatura como factor principal de la pérdida boscosa en el planeta (Gibbs et al., 2010), seguido de otros factores como la conversión a pastizales, extracción de madera, como fuente energética y para construcción, y la expansión de infraestructura (Carr, 2004). Detrás de estas causas directas se genera una serie de propuestas conceptuales sobre los motivos (subyacentes) causantes de la deforestación, que han sido categorizados en factores de tipo social, político, económico, demográfico y ambientales (Geist \& Lambin, 2001; Mather et al., 1999).

A partir de un meta-análisis de 227 estudios de deforestación tropical a nivel global, Rudel et al. (2009) identificaron que entre 1960 y 1985 las fuerzas que impulsaban la deforestación fueron de tipo social. Los resultados de este estudio mostraron un incremento en las tasas de deforestación, en donde se propusieron programas de colonización e impulsó la construcción de carreteras y nuevos asentamientos para las poblaciones rurales. A pesar de ello, la deforestación es un proceso dinámico asociado a cambios sociales, políticos y económicos y las tendencias de pérdida de bosques, desde 1985 hasta el presente, reflejan cambios en las causas y 
la influencia de procesos como la globalización, la demanda de los mercados internacionales (aumento del consumo de maíz, caña de azúcar, palma de aceite y biocombustibles) y la urbanización asociado al crecimiento poblacional urbano como impulsores más recientes de la deforestación tropical (Ramankutty et al., 2006; Rudel et al., 2009). Otros impulsores de cambio en el trópico, como se mencionaba anteriormente, se asocian con patrones generales de uso de la tierra y conversión de bosques a pastizales (Ramankutty et al., 2006).

Para América Latina, las características geográficas, los factores socio-económicos y los parámetros biofísicos han sido propuestos como los factores más importantes de cambio de uso del suelo de la región (Wassennar et al., 2007). En menor proporción e impacto, existen otros factores como la accesibilidad, demanda de mercados nacionales e internacionales y el crecimiento de la densidad poblacional, siendo las tierras bajas las fronteras de deforestación actualmente más activas (Armenteras et al., 2006, Armenteras et al., 2011, Gómez-Peralta et al., 2008, Rudel et al., 2009, Wassenaar et al., 2007).

En Latino América los estudios existentes sobre el tema reportan la deforestación de formas diferentes (deforestación total, tasa anual, tasa total, pérdida en hectáreas, etc.), que hacen que la comparación de las dinámicas de pérdida de bosque sea compleja $\mathrm{y}$, a priori, no tan directa. El objetivo de este artículo es proporcionar una revisión actualizada del conocimiento y la variabilidad regional que se presenta en la pérdida de bosques tropicales y sus causas en trece países de Latino América. Para lograr este objetivo se realizó la recopilación de información de estudios de deforestación local, regional y nacional. Posteriormente, a partir de los datos reportados se estandarizaron las tasas anuales de deforestación (Puyravaud, 2003). Adicionalmente este manuscrito presenta una recopilación de las causas subyacentes de deforestación tropical en la región y las diferencias existentes entre los países analizados.

\section{MATERIALES Y MÉTODOS}

Área de estudio: el área de estudio comprende a América Central y la parte tropical de América del Sur, excluyendo a Argentina y Chile. Países pequeños neotropicales como Belice y Cuba no fueron incluidos en el análisis debido a la poca disponibilidad de información.

Recopilación de datos: la base de datos sobre pérdida de cobertura forestal se obtuvo a partir de un análisis de casos de deforestación en la región, publicados en la literatura científica (revistas indexadas) desde el año 1990. Se realizó una búsqueda exhaustiva en tres bases de datos: a) Scopus b) Web of Knowledge y c) Google Scholar.

Los criterios de búsqueda fueron en primera instancia los años de publicación, centrando la búsqueda desde 1990 hasta 2012 (cabe aclarar que estos podían contener estudios con fechas de cobertura forestal anteriores a la fecha de publicación). Se tuvieron en cuenta las siguientes palabras clave: REDD (Reducción de Emisiones de Carbono causadas por la Deforestación y la Degradación de los Bosques), deforestation, deforestation drivers, afforestation, land use, proximate causes, deforestation causes, forest loss, tropical forest y deforestation pressures. Dichas palabras clave fueron criterios de búsqueda opcionales (OR) y no obligatorios (AND), como si lo fueron cada uno de los países. En Scopus el campo de búsqueda fue Article, Title, Abstract, Keyword. En Web of Knowledge los campos de búsqueda fueron Title y Topic. En Goggle Scholar, en la búsqueda avanzada, las palabras se ingresaron en el campo "con al menos una de las palabras" y el campo de búsqueda fue la opción "en todo el artículo".

Con los criterios de búsqueda explicados anteriormente se compilaron 283 artículos. De dicha literatura se analizó a cada uno de ellos y únicamente fueron seleccionados aquellos que reportaban la extensión inicial de bosque y la pérdida 
de área para un periodo determinado de tiempo (135 artículos). Toda la información se recopiló en una base de datos donde se registró aquella que se encontraba relacionada con la extensión, año inicial y final de bosque, así como el año inicial y final para los que el artículo reportara el proceso de deforestación. Cuando en un artículo existían varias zonas de estudio, cada una se consideró un caso aparte para ese país, ya que muchas de ellas hacían alusión a tipos de bosque diferentes. La Tabla 1 muestra el número de artículos por país y el número de datos utilizados por artículo.

Los estudios muestran datos primarios, bien sea a nivel de una región, tipo de ecosistemas o país, e identifican áreas boscosas en unidades de área (ha, $\mathrm{Km}^{2}$, etc.), tasas neta de deforestación y en algunos casos factores que los autores identificaron como contribuyentes, de alguna forma, al cambio de la cubierta forestal en sus respectivas áreas de estudio.

Estandarización de las tasas de deforestación: Para efectos de estandarización y comparación de estadísticas, con los datos de área de bosque y años se recalculó la tasa de deforestación para cada dato disponible utilizando la siguiente formula (Puyravaud, 2003):

Tasa de deforestación $=\frac{1}{\left(t_{2}-t_{1}\right)} \cdot \operatorname{Ln}\left(\frac{A_{2}}{A_{1}}\right) \cdot 100$

donde $\mathrm{A}_{1}$ y $\mathrm{A}_{2}$ son las áreas forestales en hectáreas en los años $\mathrm{t}_{1} \mathrm{y}_{2}$, respectivamente (e.g., para un periodo 1985-1990, $A_{1}$ y $A_{2}$ son los valores de cobertura forestal en 1985 y 1990, respectivamente). Estandarizar las tasas de deforestación sintetiza los resultados en términos de velocidad de cambio anual y los hace comparables entre países. De todas formas, no se debe confundir la tasa (como cambio por periodo de tiempo), con el área deforestada, aspecto que no ha sido analizado en este trabajo.

Estandarización por tipos de bosques: dadas las diferentes clasificaciones de bosque que existen en la región y dentro de cada país, los datos se sintetizaron para 5 tipos de bosques:

- Bosques de tierras bajas: agrupa los bosques húmedos tropicales (tropical forest, humid rainforest, lowland tropical forests, tropical moist forest, floodplain forest) que se encuentran por debajo de los $1000 \mathrm{msnm}$.

Tabla 1. Número de artículos seleccionados por país y número de datos de deforestación utilizados. Los años indican la fecha más antigua y más reciente para la que se reportan datos de deforestación

\begin{tabular}{lcccc}
\hline \multicolumn{1}{c}{ País } & $\begin{array}{c}\text { Número } \\
\text { artículos }\end{array}$ & $\begin{array}{c}\text { Número de datos } \\
\text { de deforestación } \\
\text { reportados }\end{array}$ & Año inicial & Año final \\
\hline Bolivia & 8 & 94 & 1956 & 2004 \\
Brasil & 41 & 119 & 1960 & 2010 \\
Colombia & 7 & 26 & 1938 & 2005 \\
Costa Rica & 3 & 21 & 1960 & 2005 \\
Ecuador & 7 & 72 & 1962 & 2002 \\
El Salvador & 1 & 3 & 1942 & 2004 \\
Guatemala & 2 & 66 & 1986 & 2003 \\
Honduras & 5 & 31 & 1955 & 2000 \\
México & 47 & 208 & 1960 & 2010 \\
Nicaragua & 3 & 11 & 1978 & 1995 \\
Panamá & 3 & 23 & 1987 & 2001 \\
Paraguay & 2 & 67 & 1989 & 2000 \\
Perú & 1 & 2 & 1987 & 2001 \\
Surinam & 1 & 2 & 2005 & 2009 \\
Venezuela & 4 & 44 & 1920 & 2008 \\
\hline
\end{tabular}


- BosqueAtlántico: corresponde a la mata atlántica $\mathrm{y}$ comprende un conjunto de formaciones como los bosques ombrófilos densos, ombrófilos mixtos, estacionales semideciduos y deciduos y ecosistemas asociados con restingas.

- Bosques secos: bosques de las regiones tropicales y subtropicales con 250 a $2000 \mathrm{~mm}$ de lluvia al año y una fuerte estación seca de al menos 3 a 4 meses (dry forest, seasonal or wetdry tropical forest).

- Bosques montanos: bosques de las zonas altas por encima de los $1000 \mathrm{msnm}$ (tropical moist montane, forest montane cloud forest, upland forest, bosque mesófilo de montaña).

- Otros: agrupa las categorías de bosques de coníferas y manglares, principalmente.

Análisis de la información: debido a la variabilidad de fechas para las que se reporta deforestación en cada uno de los países, se procedió a realizar un análisis general dirigido a la región de deforestación y otro por tipo de bosque orientado a todo el periodo de información disponible. También se analizaron las tasas para los años previos a 1990, las tasas para la década de los noventa y las tasas de deforestación más recientes, es decir del año 2000 hacia adelante.
También se recopilaron los datos oficiales de deforestación reportados por la FAO para una estadística comparativa de tasas en la región.

Las causas de deforestación fueron agrupadas en 8 grandes categorías: 1) Biofísicas, 2) Demográficas, 3) Expansión frontera agrícola, 4) Ganadería, 5) Infraestructura, principalmente presencia de carreteras, 6) Tenencia de la tierra, 7) Políticas sectoriales y 8) Otras (por ejemplo, minería, fuegos, desastres naturales) evaluando su frecuencia de aparición en cada país.

\section{RESULTADOS}

La tasa promedio anual de deforestación para toda la región durante el periodo de análisis es de -1.54, con solo tres países por arriba de esta tasa anual: Ecuador, México y Paraguay (Figura 1). Con tasas ligeramente inferiores al promedio regional, se encuentran Brasil (-1.44), Bolivia (-1.23), Venezuela (-1.18), Honduras (-1.18) y Colombia (-1.07). Los países con menores tasas de deforestación reportadas son Guatemala (-0.40), Perú (-0.30), Nicaragua (-0.26), Panamá (-0.08) y Surinam (-0.08). Dos países centroamericanos reportan recuperación de sus áreas boscosas, Costa Rica (tasas de 0.17) y El Salvador (1.87).

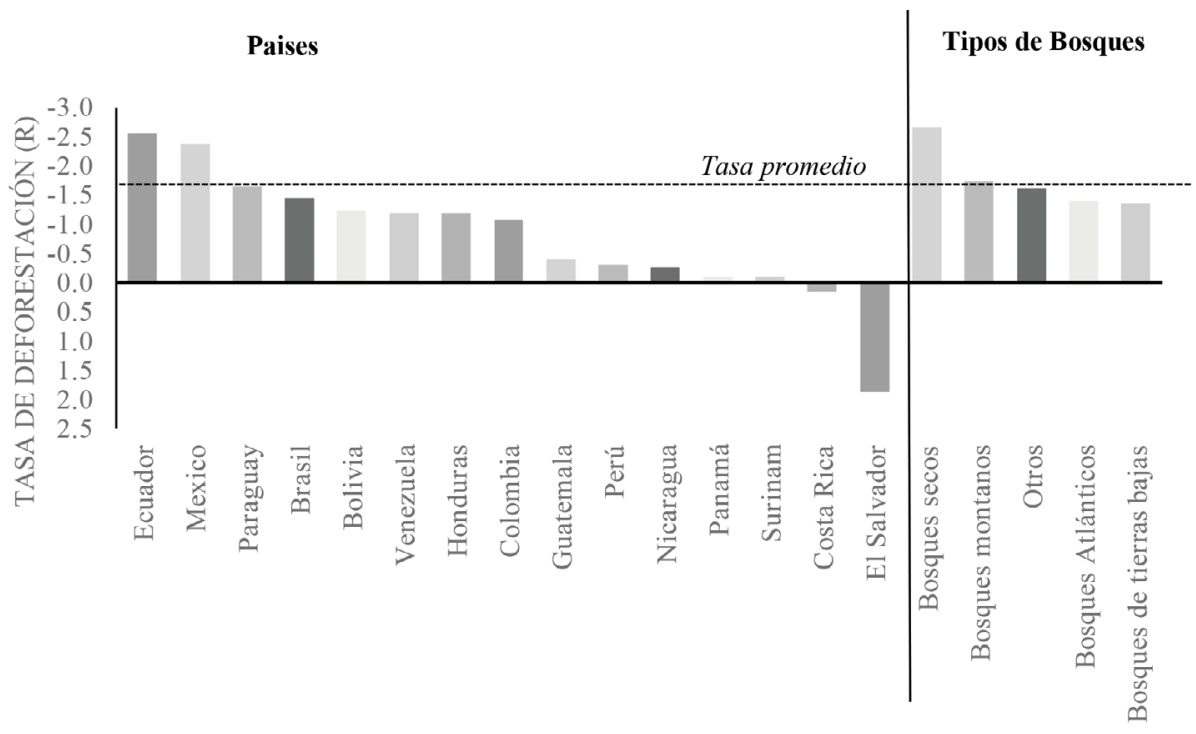

Figura 1. Tasas de deforestación por país y por tipos de bosques en Latino América 
Los bosques secos (-2.67) y los bosques montanos $(-1.72)$ presentan las tasas anuales más elevadas, seguidos por los bosques tipificados como otros (-1.61). Cuando se analizan las tasas de pérdida forestal por los tipos de bosques seleccionados (Figura 1); con una tasa ligeramente inferior al promedio se encuentran los bosques atlánticos (-1.40) seguido de los bosques de tierras bajas (-1.35) que presentan las tasas más bajas.

Al desglosar la dinámica de cambio de los diferentes tipos de bosque por país (Tabla 2), se observa un panorama ligeramente diferente, donde los resultados indican que la mayor tasa de deforestación anual corresponde a los bosques secos en México, seguida de otros tipos de bosques en Costa Rica y Brasil; adicionalmente, este último país presenta la mayor tasa de recuperación de cobertura forestal para los bosques montanos, seguida de los bosques de tierras bajas en El Salvador y de otros tipos de bosque en Panamá.

Al comparar las estadísticas de deforestación reportadas por la FAO (2010) y los datos obtenidos en este estudio (Figura 2), se observa que los promedios obtenidos a partir de la revisión de literatura son superiores en casi todos los casos a las cifras oficiales, excepto para la década de los ochenta donde se aprecia que la FAO reporta unas tasas para Centro América ligeramente superiores a las de toda la región.

Con respecto a los patrones de deforestación (Mertens \& Lambin, 1997), se observa que la mayoría de los países presentan patrones geométricos o difusos, asociados generalmente a proceso de transición hacia la consolidación de fronteras de colonización y a bosques montanos o bosques de zonas bajas donde no existen vías de acceso, la densidad de la población es baja o hay presencia de algunas figuras comunitarias de manejo de la propiedad. Solo la Amazonía brasilera y sectores de la ecuatoriana exhiben el típico patrón de espina de pescado asociado con la construcción de carreteras (Mertens \& Lambin, 1997). En algunos países se presentan patrones isla relacionados con actividad minera ilegal, generalmente de pequeña escala. El caso de Surinam evidencia dicha situación, donde esta actividad es un impulsor importante de la deforestación en la década del 2000 (Ramírez, 2001).

Tabla 2. Tasas de deforestación por tipos de bosques y por país. En negrilla indicadas las tasas de deforestación mayores $(10 \%)$ y en itálicas los cambios positivos en cobertura forestal $(10 \%)$

\begin{tabular}{llllll}
\hline Paises & $\begin{array}{l}\text { Bosques } \\
\text { Atlánticos }\end{array}$ & $\begin{array}{l}\text { Bosques de } \\
\text { tierras bajas }\end{array}$ & $\begin{array}{l}\text { Bosques } \\
\text { Montanos }\end{array}$ & Bosques Secos & Otros \\
\hline Bolivia & & -1.24 & -1.43 & -1.08 & \\
Brasil & -0.06 & -1.65 & 1.92 & -0.45 & $\mathbf{- 3 . 1 9}$ \\
Colombia & & -1.11 & -0.74 & & \\
Costa Rica & & -1.29 & & 0.99 & $\mathbf{- 4 . 8 6}$ \\
Ecuador & & -2.96 & -0.13 & & \\
El Salvador & & 1.87 & & & \\
Guatemala & & -0.40 & & & -1.31 \\
Honduras & & -0.97 & & & -1.79 \\
México & & -1.13 & -2.93 & $-\mathbf{5 . 4 4}$ & \\
Nicaragua & & -0.26 & & & \\
Panamá & & -0.41 & & -0.50 & \\
Paraguay & -1.64 & & -0.30 & & \\
Perú & & -0.08 & & & \\
Surinam & & -1.44 & & & \\
Venezuela & & & & & \\
\hline
\end{tabular}




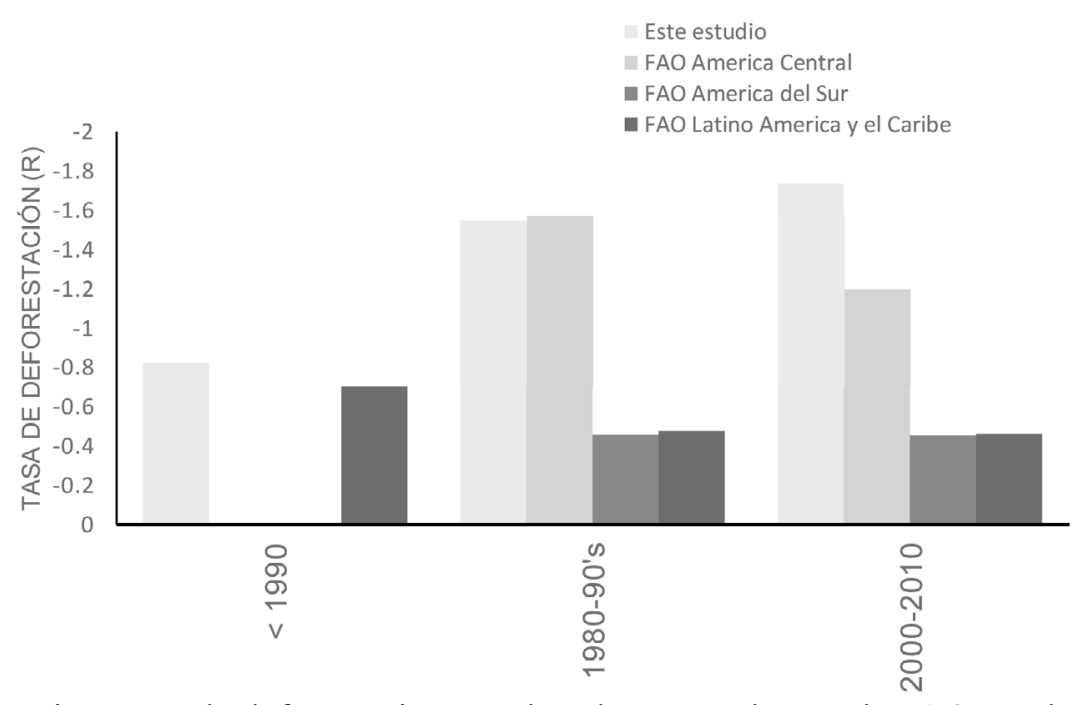

Figura 2. Comparación tasas de deforestación por década reportadas por la FAO con las tasas calculadas en este estudio para la región

El $42.5 \%$ de los estudios de caso relacionan los procesos de deforestación con la expansión de la frontera agrícola y la ganadería y un $18.85 \%$ con variables de crecimiento demográfico (Tabla 3). México y Brasil son los países con el mayor número de estudios de este tipo, seguidos de Bolivia,
Ecuador y Colombia. Guyana Francesa no reporta en sus investigaciones factores de cambio asociados y, en general, para América Central (excepto México), los impulsores de cambio se asocian a expansión agrícola y ganadera, mientras que para Sur América se asocian al crecimiento demográfico.

Tabla 3. Número de casos de estudio donde se reportan impulsores de cambio asociados a la deforestación

\begin{tabular}{|c|c|c|c|c|c|c|c|c|}
\hline País & 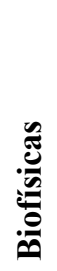 & 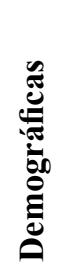 & 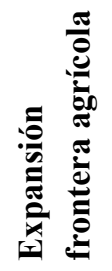 & 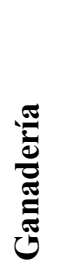 & 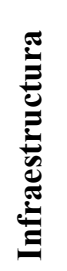 & 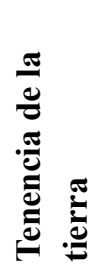 & 氖 & $\stackrel{\mathscr{0}}{0}$ \\
\hline Bolivia & 5 & 6 & 8 & 6 & 9 & 1 & 6 & \\
\hline Brasil & 4 & 15 & 29 & 27 & 15 & 8 & 4 & 2 \\
\hline Colombia & 1 & 12 & 7 & 7 & 2 & 1 & 4 & \\
\hline Costa Rica & 2 & 1 & 5 & 5 & 3 & & 2 & \\
\hline Ecuador & 3 & 7 & 5 & 3 & 7 & 4 & 7 & 1 \\
\hline El Salvador & & 1 & 2 & 1 & & & 1 & 1 \\
\hline Guatemala & 1 & 2 & 4 & 1 & 2 & & & \\
\hline Honduras & 4 & 6 & 5 & & 4 & & 1 & \\
\hline México & 9 & 23 & 34 & 20 & 15 & 4 & 20 & 4 \\
\hline Nicaragua & & 2 & 4 & & & & & 1 \\
\hline Panamá & 1 & 2 & 2 & 2 & 2 & & & \\
\hline Paraguay & 1 & 1 & 2 & 1 & 2 & 3 & 2 & 1 \\
\hline Perú & & 1 & 2 & & & & 1 & \\
\hline Surinam & & 1 & & 1 & 1 & & 2 & \\
\hline Venezuela & & 6 & 8 & 4 & & 1 & 2 & \\
\hline Total Región & 31 & 86 & 117 & 78 & 62 & 22 & 52 & 10 \\
\hline
\end{tabular}


A comienzos del año 2000 aparecen con mayor frecuencia, como agentes de la deforestación la red, empresarios y diferentes políticas sectoriales vinculadas a los hidrocarburos y los cultivos de soja, como biocombustibles, sobretodo en Brasil, Ecuador y Bolivia, así como la expansión de la infraestructura vial en la mayor parte de los países. El tema de tenencia de la tierra como una problemática asociada a los procesos de pérdida de bosques no es abordado en la mayoría de países, solo Brasil, Ecuador y México presentan análisis al respecto.

\section{DISCUSIÓN}

A pesar de la existencia de panoramas regionales (GEOLAC, 2009; FAO, 2010) y de numerosos estudios puntuales de deforestación (Tabla 4) en países de Latino América existe una alta variabilidad en las tasas reportadas, en la temporalidad y en las causas que han originado los cambios. En muchos casos hay factores vigentes que todavía actúan impulsando la deforestación en la región.

A pesar de que la gran mayoría de estudios relacionan la deforestación con la presión demográfica y la expansión de la frontera agrícola representada por cultivos a gran escala y pastizales, $\mathrm{y}$ es coincidente con lo planteado por diversos autores (Geist \& Lambin, 2001; 2002; Kaimowitz \& Angelsen, 1998; Rudel, 2007; Rudel et al., 2009; Houghton 2012; Armenteras et al., 2013), existe una relación entre la variación espacial de las tasas de deforestación y las condiciones ambientales y dinámicas socioeconómicas de cada país, las cuales responden a contextos históricos e historia del uso del suelo, existiendo aún entre los países una variabilidad de tasas de deforestación y de factores asociados, así como una variabilidad entre los tipos de bosques.

Las altas tasas de deforestación en los bosques secos y montanos donde generalmente se concentra la mayor parte de la población, y donde existen condiciones biofísicas y de infraestructuras más favorables, han estado asociados con la historia de uso del suelo desde tiempos prehispánicos y una marcada actividad económica; pese a ello, en Centroamérica los estudios de los últimos cinco años indican una tendencia positiva hacia la recuperación de bosques secundarios, fenómeno asociado al abandono de tierras por pequeños agricultores, la expansión de la agricultura comercial hacia otras áreas, el desplazamiento de la población hacia periferias urbanas y el desarrollo económico del país, como es el caso de Panamá y Costa Rica (Herrador et al., 2011; Bonilla et al., 2012; Redo et al., 2012; Aide et al., 2013).

Para los bosques bajos se estima un incremento de pastizales, así como de áreas agrícolas; el fenómeno se presenta en los frentes de colonización, sobretodo en el área del Petén (Nicaragua), Guatemala y a nivel de Suramérica en Brasil, Ecuador, Venezuela, Bolivia (Eva et al., 2004; Wassenaar et al., 2007) y el Chacó Paraguayo, producto de la interacción de fuerzas económicas y sociales complejas y disputa de la tierra (Huang et al., 2010; Chengquan et al 2011). La demanda global de biocombustibles a través del establecimiento de plantaciones de palma africana y soja, especialmente en estos países Suramericanos, son una causa reciente importante de deforestación y cambios de uso del suelo (Bonilla et al., 2012).

Aunque los bosques Atlánticos evidenciaron tasas de deforestación por debajo de la media regional, es uno de los biomas más amenazados del trópico, siendo la deforestación la principal amenaza para su biodiversidad. En Paraguay su estado es crítico, debido al cambio de uso del suelo, de forestal a agrícola, de grandes extensiones de soya, algodón y maíz (Huang et al., 2010); mientras que en Brasil se presentan diferencias regionales asociadas con diferentes estados de transición (Lira et al., 2012).

Con respecto a las causas de la deforestación, hay algunas similitudes generales en el continente. Es común en casi todos los países asociar los procesos de deforestación en las décadas de los sesenta y setenta, incluso en los ochenta (El Salvador y Nicaragua) al impulso por parte de los gobiernos de la Reforma agraria y las políticas de colonización a través del programa Alianza para el Progreso, originando diversos cambios de uso del suelo de bosques bajos, así como la apertura de vías y el 
cambio para asentamientos humanos (Rudel et al., 2009). En este sentido, el gobierno impulsó el proceso de deforestación (Rudel, 2007).

A partir de la década de los ochenta y los noventa, el establecimiento de pastizales es el factor de cambio más frecuente en América del Sur, mientras que para América Central es el establecimiento de tierras agrícolas. En países como Brasil la deforestación se vinculó con el desarrollo ganadero y agroindustrial, mientras que en el resto del continente los pequeños agricultores y colonos de las zonas bajas continuaron deforestando para establecimiento de pastizales y mejoras de la tierra (Sierra, 2000; Rudel et al., 2009). En Colombia el periodo de la bonanza del cultivo ilícito de coca (Erythroxylum coca), la falta de control estatal en su momento y el desplazamiento de la población marcó en gran parte la dinámica de la deforestación (Armenteras et al., 2011, 2013); mientras que en otros países como Nicaragua y Honduras, el conflicto armado y cambios políticos y económicos originaron áreas de ganancia de bosques (Herrador et al., 2011; Stevens et al., 2011). A partir de estos años los agentes de la mayor parte de la deforestación son empresas impulsadas por diferentes actores, bien sea grandes inversionistas o pequeños agricultores (Rudel, 2007).

Para finales de los noventa y la década del 2000 es marcada la incidencia de la globalización a partir de los beneficios de las exportaciones, el incremento de pastizales en áreas agrícolas, la mecanización de tierras para establecimiento de agricultura mecanizada y la expansión urbana (Aide \& Grau, 2004) y se evidencia que las causas de la deforestación son móviles y dependen de las fuerzas del mercado global (Lambin y Meyfroidt 2011). Actualmente los cambios de políticas y ciclos económicos aunados a la tenencia de la tierra están cambiando los agentes de la deforestación, aumentando en algunos países el impacto de los pequeños y medianos agricultores (Killeen et al., 2008); y aun cuando existen iniciativas como los programas de Reducción de Emisiones de Carbono causadas por la Deforestación y la Degradación de bosques (REDD)+, hasta la fecha su implementación no ha sido evaluada a nivel del continente. Sin duda uno de los aspectos a monitorear será la eficiencia de este tipo de programas en reducir las tasas de deforestación en los países de la región.

Los efectos de las carreteras han tenido una correlación positiva con la deforestación en la mayoría de los casos, salvo países que no cuentan con infraestructura vial en bosques de tierras bajas, ya que son los ríos los que cumplen este papel (Armenteras et al., 2006; Soares -Filho et al., 2004). Peres \& Schneider (2012), indican que la población de la Amazonía brasilera creció en un $225 \%$ desde 1970, donde el establecimiento de asentamientos humanos en los estados de Pará y Acre, son de aproximadamente a 10.6 millones de hectáreas de bosque deforestados hasta el 2004.

Los resultados de este trabajo se basan en datos provenientes de diferentes fuentes que han sido agregados y que presentan diferente cubrimiento por país a diferentes escalas, pero que permiten desplegar y analizar un panorama regional y una primera aproximación a la variabilidad regional presente en Latino América. Se espera que sobre la base de posteriores investigaciones científicas, la actualización de los datos nacionales, reportes de la FAO y otros estudios que se generen en el futuro cercano, se tenga mayor información que mejore los datos de forma homogénea y comparable entre los países. Finalmente, es necesario continuar con el monitoreo de la deforestación a fin de complementar los resultados que deben cumplir a nivel mundial los países para reducir las emisiones de gases de efecto invernadero por deforestación a través de estrategias como REDD+. 


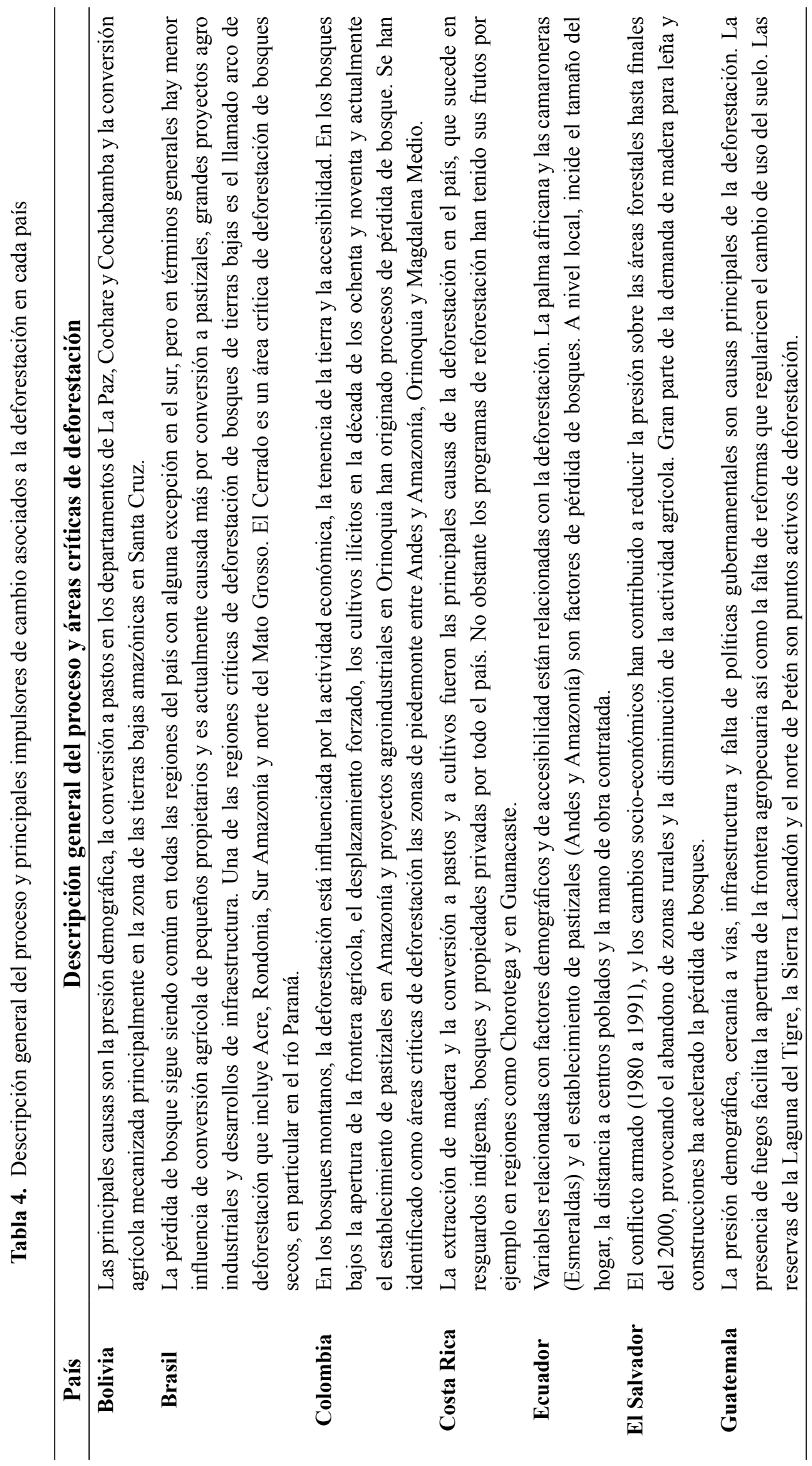


Dolor Armenteras / Nelly Rodríguez E.

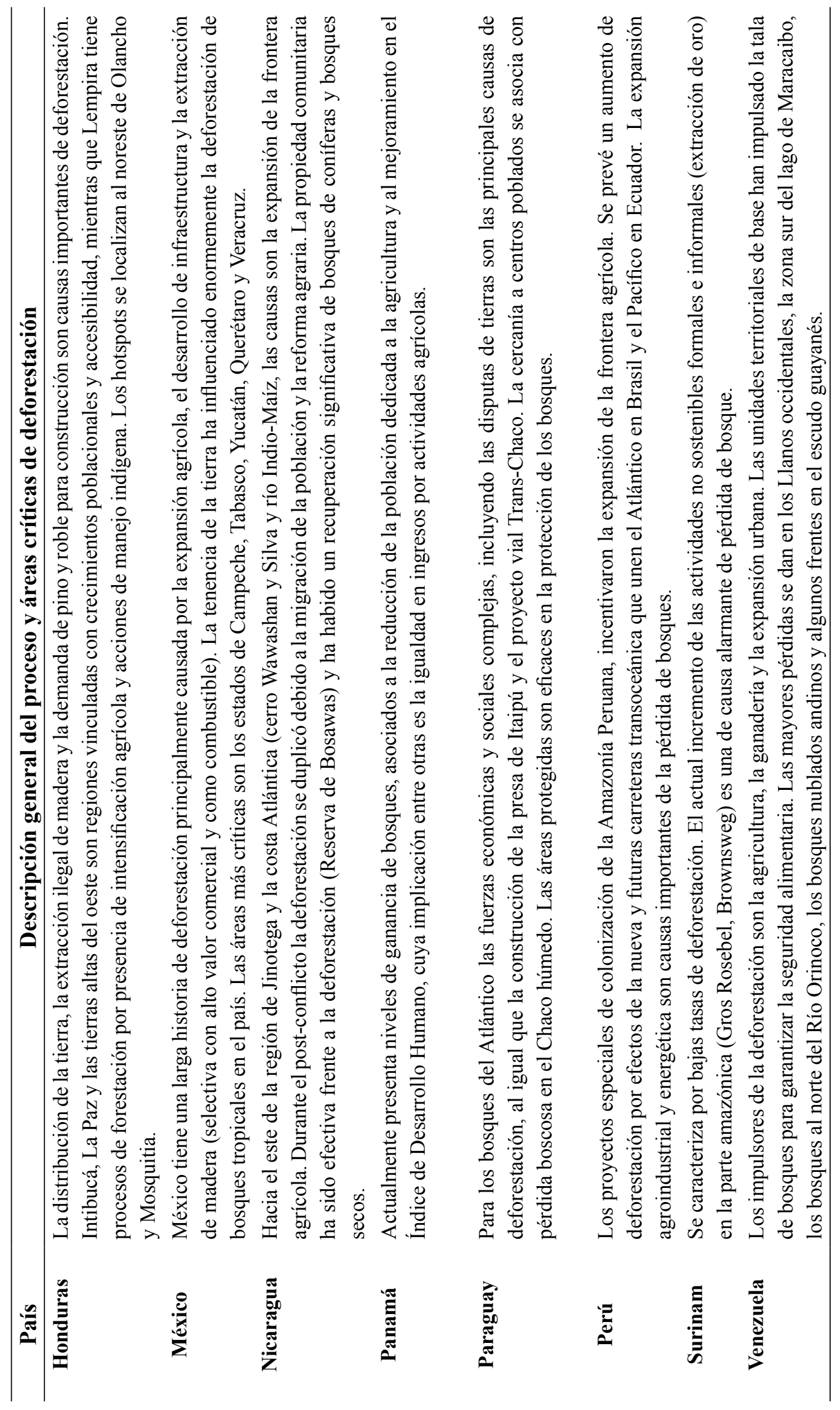




\section{AGRADECIMIENTOS}

Queremos agradecer a los miembros del grupo de Ecología del Paisaje y Modelación de Ecosistemas que apoyaron la búsqueda de información, en particular a Eduardo Molina, Jorge Paiba y María Alejandra Chadid, así como a las estudiantes de pregrado Diana Monroy y Ángela Mayorga. Agradecemos el apoyo financiero de la Universidad Nacional de Colombia con su convocatoria de Fortalecimiento de Alianzas estratégicas interinstitucionales en el marco de la alianza y Red Cyted para el monitoreo del estado de la conservación y recuperación de bosques húmedos y secos en Latino América en el contexto de la deforestación evitada, con el código proyecto: Red CYTED (P411RT0559).

\section{REFERENCIAS BIBLIOGRÁFICAS}

Achard, F., Eva, H.D., Stibig, H.-J., Mayaux, P., Gallego, J., Richards, T. \& Malingreau, J.-P. (2002). Determination of deforestation rates of the world's humid tropical forests. Science, 297, 999-1002.

Aide, T. M. \& Grau, H. R. (2004). Globalization, migration and Latin American ecosystems. Science, 305, 1915-1916.

Aide, T.M., Clark, M.L., Grau, H.R., LópezCarr, D., Levy, M. a., Redo, D., BonillaMoheno, M., Riner, G., Andrade-Núñez, M.J., Muñiz, M. (2013). Deforestation and Reforestation of Latin America and the Caribbean (2001-2010). Biotropica, 45, 262-271.

Armenteras, D., Rudas, G., Rodríguez, N., Sua, S., \& Romero, M. (2006). Patterns and causes of deforestation in the Colombian Amazon. Ecological Indicators, 6(2), 353368.

Armenteras, D., Rodríguez, N., Retana, J., \& Morales, M. (2011). Understanding deforestation in montane and lowland forests of the Colombian Andes. Regional Environmental Change, 11(3), 693-705.

Armenteras, D., Cabrera, E., Rodríguez, N., \& Retana, J. (2013). National versus and regional determinants of tropical deforestation: a case study of in Colombia. Regional Environmental Change, 13 (6), 1181-1193.

Asner, G.P., Rudel, T.K., Aide, T.M., Defries, R. \& Emerson, R. (2009). A contemporary assessment of change in humid tropical forests. Conservation Biology, 23, 1386-95.

Bonilla-Moheno, M., Aide, T.M., Clark, M.L. (2012). The influence of socioeconomic, environmental, and demographic factors on municipality-scale land-cover change in Mexico. Regional Environmental Change, 12, 543-557.

Carr, D. (2004). Proximate population factors and deforestation in tropical agricultural frontiers. Population and Environment, 25 (6), 585-612

Chengquan, H., Kim, Sunghee, K., Townshend, K., Davis, J.R.G., Altstatt, P., Rodas, A., Yanosky, O., Clay, A., Tucker, R., Compton, J. \& Musinsky, J. (2011). Assessment of Paraguay's forest cover change using Landsat observations. Global and Planetary Change, 1-2, 1-12.

Eva, H.D., Belward, A.S., De Miranda, E.E., Di Bella, C.M., Gond, V., Huber, O., Jones, S., Sgrenzaroli, M., Fritz, S. (2004). A land cover map of South America. Global Change Biology, 10, 731-744.

Food and Agriculture Organization of the United Nations (FAO) (2010). Global Forest Resource Assessment. Rome: FAO.

Food and Agriculture Organization of the United Nations (FAO) (2011). State of the World's Forests. Chapter 2. Rome: FAO. 
FRA (2010). Evaluación de los recursos forestales mundiales 2010. Estudio Montes, 163. Roma: FAO.

Geist, H.J. \& Lambin, E.F. (2001). What drives tropical deforestation? A meta-analysis of proximate and underlying causes of deforestation based on subnational case study evidence. LUCC Report Series 4, CIACO, Louvain-la-NeuveBelgium.

Geist, H.J. \& Lambin, E.F. (2002). Proximate causes and underlying driving forces of tropical deforestation. BioScience, 52, 143-150.

GEOLAC (2009). Perspectivas del medio ambiente: América Latina y el Caribe. Programa de las Naciones Unidas para el Medio Ambiente. Oficina Regional para América Latina y el Caribe. Panamá: FAO.

Gibbs, H.K., Ruesch, A.S., Achard, F., Clayton, M.K., Holmgren, P., Ramankutty, N.\& Foley, J. A. (2010). Tropical forests were the primary sources of new agricultural land in the 1980s and 1990s. Proceedings of the National Academy of Sciences, 107, 16732-7.

Gomez-Peralta, D., Oberbauer, S.F., McClain, M.E., Philippi, T.E. (2008). Rainfall and cloud-water interception in tropical montane forests in the eastern Andes of Central Peru. Forest Ecology and Management, 255, 1315-1325.

Hansen, M.C., Stehman, S. V. \& Potapov, P. V. (2010). Quantification of global gross forest cover loss. Proceedings of the National Academy of Sciences, 107, 8650-5.

Herrador, D., Boada i Juncà, M., Varga, D. \& Mendizábal, E. (2011). Tropical forest recovery and socio-economic change in El Salvador: An opportunity for the introduction of new approaches to biodiversity protection. Applied Geography, 1, 259-268.

Houghton, R. (2012). Carbon emissions and the drivers of deforestation and forest degradation in the tropics. Current Opinions in Environmental Sustainability, 4, 597-603.

Huang, C., Goward, S. N., Masek, J. G., Thomas, N., Zhu, Z., \& Vogelmann, J. E. (2010). An automated approach for reconstructing recent forest disturbance history using dense Landsat time series stacks. Remote Sensing of Environment, 114(1), 183-198.

Kaimowitz, D. \& Angelsen, A. (1998). Economic models of tropical deforestation: A review. Centre for International Forestry Research. Jakarta: Cifor. $139 \mathrm{p}$.

Killeen, T., A. Guerra, A., Calzada, M., Correa, L., Calderon, V., Soria, L., Quezada, B. \& Steininger, M. (2008). Total historical land use change in eastern Bolivia: who, where, when, and how much. Ecology and Society, 13, 16-27.

Mather, A.S., Needle, C.L. \& Fairbairn, J. (1999). Environmental Kuznets Curves and Forest Trends. Geography, 84, 55-65.

Mertens, B., \& E. Lambin. (1997). Spatial modelling of deforestation in southern Cameroon. Spatial disaggragation of diverse deforestation processes. Applied Geography, 17, 143-162.

Lambin, E.F., \& P. Meyfroidt. (2011). Global land use change, economic globalization, and the looming land scarcity. Proceedings of the National Academy of Sciences, 108, 3465-3472.

Lira, P.K., Tambosi, L.R., Ewers, R.M. \& Metzger, J.P. (2012). Land-use and landcover change in Atlantic Forest landscapes. 
Forest Ecology and Management, 278, 8089.

Peres, C. A. \& Schneider, M. (2012). Subsidized agricultural resettlements as drivers of tropical deforestation. Biological Conservation, 151, 65-68.

Puyravaud, J.P. (2003). Standardizing the calculation of the annual rate of deforestation. Forest Ecology and Management, 177, 593-596.

Ramankutty, N., Graumlich, L., Achard, F., Alves, D., Chhabra, A., Defries, R.S., Foley, J., Geist, H., Houghton, R.A., Goldewijk, K.K., Lambin, E.F., Millington, A., Rasmussen, K., Reid, R. \& Turner, B.L. (2006). Global landcover change: recent progress, remaining challenges (Chapter 2). In: Lambin, E.F., Geist, H. (Eds.). Land-Use and LandCover Change. Local Processes and Global Impacts. Springer, pp. 9-39.

Ramírez, S.O. (2011). Spatial drivers of deforestation in Suriname. Center for agriculture research in Suriname. Paramaribo: Tropenbos. $42 \mathrm{p}$.

Redo, D.J., Grau, H.R., Aide, T, M. \& Clark, M. (2012). Asymmetric forest transition driven by the interaction of socioeconomic development and environmental heterogeneity in Central America. Proceedings of the National Academy of Sciences of the United States of America, 109, 8839-44.
Rudel, T.K. (2007). Changing agents of deforestation: From state-initiated to enterprise driven processes, 1970-2000. Land Use Policy, 24, 35-41.

Rudel, T.K., Defries, R., Asner, G.P. \& Laurance, W.F. (2009). Changing drivers of deforestation and new opportunities for conservation. Conservation Biology, 23, 1396-405.

Sierra, R. (2000). Dynamics and patterns of deforestation in the western Amazon: the Napo deforestation front, 1986-1996. Applied Geography, 20, 1-16.

Soares-Filho, B., Alencar, A., Nepstad, D., Cerqueira, G., Vera Diaz, M. D. C., Rivero, S., Voll, E. (2004). Simulating the response of land-cover changes to road paving and governance along a major Amazon highway: the Santarem-Cuiaba corridor. Global Change Biology, 10(5), 745-764.

Stevens, K., Campbell, L., Kramer, G. \& Jiaguo, D. (2011). Examining complexities of forest cover change during armed conflict on Nicaragua's Atlantic Coast. Biodiversity and Conservation, 12, 2597-2613.

Wassenaar,T., Gerber, P., Verburg, P.H., Rosales, M., Ibrahim, M. \& Steinfeld H (2007). Projecting land use changes in the Neotropics: The geography of pasture expansion into forest. Global Environmental Change, 17, 86-104. 